\title{
Rescue of gene expression by modified REST decoy oligonucleotides in a cellular model of Huntington's disease
}

Article

Published Version

Open Access (Wiley Online Open)

Soldati, C., Bithell, A., Conforti, P., Cattaneo, E. and Buckley, N. J. (2011) Rescue of gene expression by modified REST decoy oligonucleotides in a cellular model of Huntington's disease. Journal of Neurochemistry, 116 (3). pp. 415-425. ISSN 0022-3042 doi: https://doi.org/10.1111/j.14714159.2010.07122.x Available at https://centaur.reading.ac.uk/34707/

It is advisable to refer to the publisher's version if you intend to cite from the work. See Guidance on citing.

To link to this article DOI: http://dx.doi.org/10.1111/j.1471-4159.2010.07122.x

Publisher: Wiley-Blackwell

All outputs in CentAUR are protected by Intellectual Property Rights law, including copyright law. Copyright and IPR is retained by the creators or other copyright holders. Terms and conditions for use of this material are defined in the End User Agreement. 


\section{CentAUR}

Central Archive at the University of Reading

Reading's research outputs online 


\title{
Rescue of gene expression by modified REST decoy oligonucleotides in a cellular model of Huntington's disease
}

\author{
Chiara Soldati,* Angela Bithell,* Paola Conforti, $\dagger$ Elena Cattaneo $\dagger$ and Noel J. Buckley* \\ *Department of Neuroscience and Centre for the Cellular Basis of Behaviour, Institute of Psychiatry, King's College London, \\ The James Black Centre, London, UK \\ $\dagger$ Department of Pharmacological Sciences and Centre for Stem Cell Research, University of Milano, Milan, Italy
}

\begin{abstract}
Transcriptional dysfunction is a prominent hallmark of Huntington's disease (HD). Several transcription factors have been implicated in the aetiology of HD progression and one of the most prominent is repressor element 1 (RE1) silencing transcription factor (REST). REST is a global repressor of neuronal gene expression and in the presence of mutant Huntingtin increased nuclear REST levels lead to elevated RE1 occupancy and a concomitant increase in target gene repression, including brain-derived neurotrophic factor. It is of great interest to devise strategies to reverse transcriptional dysregulation caused by increased nuclear REST and determine the consequences in HD. Thus far, such strategies have involved RNAi or mutant REST constructs. Decoys are double-stranded oligodeoxynucleotides corresponding to the
\end{abstract}

DNA-binding element of a transcription factor and act to sequester it, thereby abrogating its transcriptional activity. Here, we report the use of a novel decoy strategy to rescue REST target gene expression in a cellular model of HD. We show that delivery of the decoy in cells expressing mutant Huntingtin leads to its specific interaction with REST, a reduction in REST occupancy of RE1s and rescue of target gene expression, including Bdnf. These data point to an alternative strategy for rebalancing the transcriptional dysregulation in HD.

Keywords: brain-derived neurotrophic factor, decoy oligonucleotides, histone modification, Huntington's disease, repressor element 1 silencing transcription factor, transcriptional dysregulation.

J. Neurochem. (2011) 116, 415-425.
Huntington's disease (HD) is an autosomal-dominant disorder characterised by neurodegeneration that leads to irrepressible motor dysfunction, cognitive decline and psychiatric disturbances, progressive dementia and death approximately 20 years after disease appearance (Zuccato et al. 2010). HD results from an aberrantly expanded repetition of a CAG nucleotide triplet in the huntingtin (Htt) gene that produces a polyglutamine extension at the $\mathrm{N}$ terminus of the Htt protein (Huntington's Disease Collaborative Research Group 1993; Bates 2005). Although the abnormal protein is ubiquitously expressed, cell degeneration occurs mainly in the brain. Over the course of HD, the degeneration of GABA-releasing spiny projection neurons in the striatum is predominant; however, loss of neurons in other brain regions, such as cerebral cortex has also been reported (Zuccato et al. 2010). Htt is a large multi-domain protein, whose function is still not fully understood but is thought to have multiple distinct cellular roles, including intracellular transport, synaptic function, anti-apoptotic activity and transcriptional regulation (Cattaneo et al.
2005). However, much attention has focussed on the transcriptional dysregulation that occurs in the presence of mutant $\mathrm{Htt}$ (muHtt). Both wild-type and muHtt can interact with a number of transcription factors such as cAMPresponse element binding protein-binding protein (Steffan et al. 2000; Nucifora et al. 2001), TATA-binding protein

Received August 31, 2010; revised manuscript received November 11, 2010; accepted November 12, 2010.

Address correspondence and reprint requests to Prof. Noel J. Buckley, Department of Neuroscience and Centre for the Cellular Basis of Behaviour, Institute of Psychiatry, King's College London, The James Black Centre, 125 Coldharbour Lane, London SE5 9NU, UK.

E-mail: noel.buckley@kcl.ac.uk

Abbreviations used: Bdnf, brain-derived neurotrophic factor; ChIP, chromatin immunoprecipitation; Chrm4, M4 muscarinic receptor gene; DN, dominant-negative; EMSA, electrophoretic mobility shift assay; H3K9ac, histone H3 acetyl-lysine 9; HD, Huntington's disease; HDAC, histone deacetylase; Htt, Huntingtin; muHtt, mutant Huntingtin; NSCs, neural stem cells; ODN, double-stranded oligonucleotide decoy; qPCR, quantitative PCR; RE1, repressor element 1; REST, RE1 silencing transcription factor; Syn1, synapsin 1 . 
(Huang et al. 1998), Sp1 (Dunah et al. 2002), p53 (Steffan et al. 2000; Bae et al. 2005), transcription initiation factor TFIID $130 \mathrm{kDa}$ subunit (Dunah et al. 2002) and TATAbinding protein (Huang et al. 1998) and these interactions are frequently disrupted in the presence of muHtt (Rubinsztein and Carmichael 2003). Recent work has highlighted repressor element 1 (RE1) silencing transcription factor (REST) (also known as neuron-restrictive silencing factor) as one transcription factor, whose function is disrupted in HD (Zuccato et al. 2003, 2007; Bithell et al. 2009; Johnson and Buckley 2009). REST was first identified as a regulator of neuronal genes in non-neuronal cells (Kraner et al. 1992; Mori et al. 1992; Schoenherr and Anderson 1995). Genome-wide chromatin immunoprecipitation (ChIP) and bioinformatic studies have subsequently revealed several thousand genomic REST binding loci (RE1 sites), which are linked to genes that encode non-neuronal as well as neuronal proteins (Bruce et al. 2004; Johnson et al. 2006, 2007, 2008a). Further, REST regulates target genes in both neural progenitors (Johnson et al. 2008a) and in mature neurons and glia (Palm et al. 1998; Abrajano et al. 2009a,b). Indeed REST dysfunction in neurons has been implicated in several neurological and neurodegenerative pathologies, including epileptic seizure (Palm et al. 1998; Garriga-Canut et al. 2006; Spencer et al. 2006), ischaemia (Calderone et al. 2003) and HD (Zuccato et al. 2003, 2007; Buckley et al. 2010).

Previous work has shown that wild-type $\mathrm{Htt}$ can sequester REST in the cytoplasm whereas in the presence of muHtt, this association is disrupted and leads to increased levels of nuclear REST resulting in increased REST occupancy of RE1 sites and repression of target genes (Zuccato et al. 2007). One key REST target gene that is repressed in the presence of muHtt is $B d n f$. Brain-derived neurotrophic factor (BDNF) is supplied by cortical projections to the striatum where it is necessary for the maintenance and survival of medium spiny projection neurons, the most prominent neuronal population that dies during HD (Altar et al. 1997; Canals et al. 2004; Zala et al. 2005; Zuccato and Cattaneo 2009). Several studies have shown reduced BDNF levels in both animal and cellular models of HD. These studies indicate that a decrease in cortical BDNF occurs early in the disease process and is followed by a progressive decline, strongly implicating the role of this neurotrophin in HD pathology (Zuccato et al. 2001, 2007, 2008). REST occupancy of RE1 sites, including at the $B d n f$ promoter, is increased in HD models including $H d h^{109 / 109}$ knock-in and R6/2 mice (Zuccato et al. 2007). Importantly, in the case of $H d h^{109 / 109}$ mice, this increased occupancy precedes the development of a neuropathological phenotype (Zuccato et al. 2007). This suggests that dysregulation of REST target gene expression might be an early molecular event in HD and thus attenuating REST binding during early disease stages could be of therapeutic benefit.
Subsequently, we have shown that use of a dominantnegative REST construct, comprising only the eight zinc finger DNA-binding domain and lacking any co-repressor interaction domains, can rescue BDNF levels (mRNA and protein) in cellular models of HD (Zuccato et al. 2007). Although such mutant constructs are useful tools, they are large and require viral delivery for optimal results. An alternative strategy is deployment of decoy oligodeoxynucleotides (ODNs) that act as surrogate binding sites for transcription factors and sequester the native transcription factor from its genomic binding sites. Decoy ODNs have been used successfully to inhibit the binding and transcriptional action of several transcription factors including nuclear factor-kappaB protein, $\mathrm{Sp} 1$, activator protein 1, signal transducer and activator of transcription $1 / 3$ and nuclear factor of activated T-cells 1c (Griesenbach et al. 2002; Chae et al. 2006; Stadlbauer et al. 2008). Furthermore, this approach has been used to successfully deliver and attenuate transcription factor function in neural cells in culture (Steiger et al. 2004; Meller et al. 2005; Yao et al. 2005; AharoniSimon et al. 2006), in brain slice preparations and in vivo (Ye and Johnson 2001; Kubo et al. 2003; Dash et al. 2005). The main limitation of unmodified ODNs is their susceptibility to degradation by nucleases prevalent in sera and cells. To rectify this problem, ODNs with modified linkages, such as phosphorothiolate and methylphosphonate, have been developed (Lee et al. 2003; Osako et al. 2007). Here, we describe the development of modified decoy ODNs to inhibit RESTmediated gene repression in a cellular model of HD and show efficient rescue of aberrant gene expression.

\section{Methods}

$\mathrm{Hdh}^{109 / 109}$ and $\mathrm{Hdh}^{7 / 7}$ cell culture and transfection

$H d h^{7 / 7}$ and $H d h^{109 / 109}$ cell lines were previously generated from the striata of wild-type and homozygous knock-in mice with each Htt allele bearing 7 or 109 CAG repeats, respectively, as described by Trettel et al. (2000). Both cell lines were propagated in Dulbecco's modified Eagle's medium supplemented with $10 \%$ foetal calf serum at $33^{\circ} \mathrm{C}$ in a humidified $5 \% \mathrm{CO}_{2}$ atmosphere. Transfection with ICAFectin (Eurogentec, Southampton, UK) was performed according to manufacturer's instructions and optimal transfection efficiency $(50-60 \%)$ was achieved using $0.5 \mu \mathrm{g}$ of $\mathrm{DNA} / \mu \mathrm{L}$ of ICAFectin. Transfections were carried out in the presence of serum and medium was replaced after 12-24 h.

\section{Construction of phosphorothiolated RE1 decoy ODN}

Single stranded oligonucleotides were synthesised by Sigma Genosys (St. Louis, MO, USA). Annealing was performed in 10× annealing buffer (100 mм Tris-HCl, pH8.0, $10 \mathrm{~mm}$ EDTA, $1 \mathrm{~m}$ $\mathrm{NaCl})$ by heating to at least $5-10^{\circ} \mathrm{C}$ above their $T_{\mathrm{m}}$ and cooling slowly to $4^{\circ} \mathrm{C}$ over $3-4 \mathrm{~h}$ using a heatblock. A RE1 decoy $(++\mathrm{D})$ was designed corresponding to a canonical REST-binding site (Johnson et al. 2006). A negative decoy control (--D) was generated using a sequence corresponding to a non-canonical RE1 
that has been shown not to bind REST (Bruce et al. 2004). The decoy ODN sequences, including a phosphorothiolate modification on the first three nucleotides were: Positive decoy $(++\mathrm{D})$ : $5^{\prime}-G^{\mathrm{p}} \mathrm{P}^{\mathrm{p}} \mathrm{C}^{\mathrm{p}} \mathrm{PTPTTCAGCACCACGGACAGCGCCAGC-3}$; ; Negative decoy (--D): $5^{\prime}-G^{p} P^{p} C^{p} P T P T C C A G C A C A G T G G T C A G A-$ CCC-3'.

\section{DNA binding and electromobility shift assay (EMSA)}

For gel shift analysis, $12.5 \mathrm{~nm}$ of infrared (IR700) fluorescencelabelled decoy (IR-Decoy) was incubate with $0.25 \mu \mathrm{g}$ HeLa cell nuclear extract in binding buffer $(40 \mathrm{~mm}$ Tris- $\mathrm{HCl}, \mathrm{pH} 8,0.4 \mathrm{mg} / \mathrm{mL}$ bovine serum albumin, $200 \mathrm{~nm} \mathrm{ZnCl}_{2}, 400 \mathrm{~mm} \mathrm{KCl}, 2 \mathrm{~mm} \mathrm{MgCl}$, $40 \%$ glycerol, $0.4 \%$ NP-40, Sigma, St. Louis, MO, USA) and $2 \mathrm{~mm}$ $\beta$-mercaptoethanol for $1 \mathrm{~h}$ at $4^{\circ} \mathrm{C}$ in the dark. For supershift experiments, $2 \mu \mathrm{L}$ of anti-REST rabbit polyclonal (P-18X, $2 \mathrm{mg}$ / $\mathrm{mL}$; Santa Cruz Biotechnology, Santa Cruz, CA, USA) was added to the mixture. For excess negative decoy assays, $50 \mathrm{~nm}$ final concentration of negative decoy (--D) was added to the mixture. Reaction mixtures were separated on a $4 \%$ non-denaturing polyacrylamide gel at $4^{\circ} \mathrm{C}$ in $0.5 \times \mathrm{TBE}(90 \mathrm{~mm}$ Tris borate, $2 \mathrm{~mm}$ EDTA, pH8.3) at $150 \mathrm{~V}$ for $2 \mathrm{~h}$ at $4^{\circ} \mathrm{C}$ in the dark. The gel was prerun at $150 \mathrm{~V}$ for $30 \mathrm{~min}$ at $4^{\circ} \mathrm{C}$. Gels were scanned with an Odyssey Infrared Imaging System (Li-cor, NE, USA). The decoy ODN sequences used, including 5'-/5IRD700 modifications, were 5'-/ 5IRD700/TTCAGCACCACGGACAGCGCC-3' and 5'-/5IRD700/ GGCGCTGTCCGTGGTGCTGAA-3' (Integrated DNA Technologies, Belgium).

\section{RNA extraction and cDNA synthesis}

Total RNA was extracted from $H d h^{109 / 109}$ and $H d h^{7 / 7}$ cells using Trizol (Invitrogen, Carlsbad, CA, USA) and digested with DNAse I (Ambion, Austin, TX, USA). For each RNA sample, $2 \mu \mathrm{g}$ of total RNA was reverse transcribed for $60 \mathrm{~min}$ at $37^{\circ} \mathrm{C}$ with random hexamers (Promega, Madison, WI, USA) and Moloney murine leukemia virus reverse transcriptase (Promega, WI, USA).

\section{Real-time PCR and quantification of gene expression}

Polymerase chain reactions were run with the following condition: $95^{\circ} \mathrm{C}$ for $3 \mathrm{~min}$, then 45 cycles $\times\left(30 \mathrm{~s} 95^{\circ} \mathrm{C}, 30 \mathrm{~s} 60^{\circ} \mathrm{C}, 30 \mathrm{~s}\right.$ $72^{\circ} \mathrm{C}$ ). Real-time PCR was performed using iQ SYBR Green Supermix (Bio-Rad Laboratories, Hercules, CA, USA) with $0.5 \mu \mathrm{M}$ primers. Expression levels were analysed according to the Pfaffl method (Pfaffl et al. 2002) and then normalised to levels of Gapdh. Error bars show the standard deviations obtained from two samples run in triplicate in two different assays. Primer sequences - Gapdh (Fwd: 5'-TCCGACTTCAACAGCAACTC-3'; Rev: 5'-CTTGCTCAGTGTCCTTGCTG-3'); REST (Fwd: 5'-CGAACTCACACAGGAGAACG-3'; Rev: 5'-GAGGCCACATAATTGCACTG-3'); M4 muscarinic receptor gene (Chrm4) (Fwd: 5'-TCCTCACCTGGACACCCTAC-3'; Rev: 5'-ACGTAGCAGAGCCAGTAGCC-3'); Bdnf (Fwd: 5'-TCGTTCCTTTCGAGTTAGCC-3'; Rev: 5'-TTGGTAAACGGCACAAAACA-3'); synapsin 1 (Syn1) (Fwd: 5'-GAGCAGATTGCCATGTCTGA-3'; Rev: 5'-CACTGCGCAGATGTCAAGTC-3').

\section{Chromatin immunoprecipitation}

Cells were fixed in $1 \%$ formaldehyde at $20-25^{\circ} \mathrm{C}$ for $5 \mathrm{~min}$ before washing in cold phosphate-buffered saline and centrifugation at
$2200 \mathrm{~g}$ for $5 \mathrm{~min}$. Cells were lysed in lysis buffer for $30 \mathrm{~min}$ on ice, cell nuclei pelleted and resuspended in shearing buffer before sonication in a Bioruptor (Diagenode, Belgium) to obtain an average chromatin shear size of 200-500 bp. All steps included protease inhibitor cocktail (Roche Molecular Biochemicals, Indianapolis, IN, USA). Pre-cleared chromatin $(20 \mu \mathrm{g})$ was immunoprecipitated with 1-2 $\mu \mathrm{g}$ of antibody at $4^{\circ} \mathrm{C}$ for $3 \mathrm{~h}$ and $25 \mu \mathrm{L}$ magnetic protein-G beads (Active Motif, Carlsbad, CA, USA) was added for $1 \mathrm{~h}$ at $4^{\circ} \mathrm{C}$ before magnetic capture. Following a series of washes, eluted chromatin was de-crosslinked, proteinase K-treated and ChIP DNA was purified using QIAquick PCR purification kit (Qiagen, Valencia, CA, USA). Eluted ChIP DNA was analysed using quantitative PCR (qPCR). An anti-REST rabbit polyclonal antibody (Millipore Corporation, Bedford, MA, USA) was used to precipitate chromatin. Non-specific binding was assessed by immunoprecipitation with non-immune rabbit IgG and enrichments were normalised to those at the Chrm 4 coding region, a locus greater than $10 \mathrm{~kb}$ distal to any known RE1 site. RE1 flanking primer sequences were as follows: Bdnf RE1 [Fwd: 5'-GGATTTGTCCGAGGTGGTAG3'; Rev: 5'-CGGAAAAGACGCTTTTTAAGG-3' (these primers are designed to amplify exon 2, present in all Bdnf transcripts)]; Syn1 RE1 (Fwd: 5'-GCACACTCAGAGGGGAACAT-3'; Rev: 5'-CTCTTTTCTTTGCCCGACAG-3'); Chrm4 RE1 (Fwd: 5'-AGGAGACTCGTGCCATCTTC-3'; Rev: 5'-CGAGCATCTACCCTGGCTAA-3 ${ }^{\prime}$.

\section{Immunocytochemistry}

Cells were fixed for $10 \mathrm{~min}$ in $4 \%$ paraformaldehyde, permeabilised in $0.1 \%$ Triton X-100 (Sigma) for 5 min then incubated with antiREST ( 1 : 200; Upstate Biotechnology, Lake Placid, NY, USA) for $1 \mathrm{~h}$ at $20-25^{\circ} \mathrm{C}$ in phosphate-buffered saline containing $10 \%$ normal goat serum. Secondary antibody incubation was performed for $30 \mathrm{~min}$ at $20-25^{\circ} \mathrm{C}$ with $\mathrm{Cy} 5$-conjugated rabbit anti-goat IgG (1: 500; Jackson Labs, CA, USA). Nuclei were counter-stained with 4',6-diamidino-2-phenylindole (DAPI) and coverslips were mounted in Floromount-G (Southern Biotech, AL, USA).

\section{BDNF ELISA assays}

Cell lysates were prepared in lysis buffer consisting of $10 \%$ glycerol, 25 mm Tris HCl, pH 7.5, 150 mm NaCl, 1\% Triton X-100, 5 mm EDTA and 1 mм EGTA supplemented with $1: 100$ Protease Inhibitor Mixture (Sigma-Aldrich, St Louis, MO, USA). Samples were homogenised, sonicated and centrifuged at maximum speed for $15 \mathrm{~min}$ at $4^{\circ} \mathrm{C}$. The supernatants were collected and stored at $-30^{\circ} \mathrm{C}$. Samples were assayed for BDNF using the ImmunoAssay System (Promega) according to manufacturer's instructions.

\section{Nuclear and cytoplasmic western blotting}

Cytoplasmic and nuclear protein fractions were extracted using NEPER Nuclear and Cytoplasmic extraction reagents (Thermo scientific, MA, USA) with protease inhibitors (Protease Inhibitor Cocktail; Roche) according to manufacturer's instructions. Protein concentrations were determined using a Bicinchoninic Acid Kit (Sigma) and a Tecan plate reader with Magellan5 software (Tecan, Switzerland). Fifty microgram of nuclear or cytoplasmic protein for each sample was boiled for $5 \mathrm{~min}$ in Laemmli sample buffer and run on $6 \%$ sodium dodecyl sulphate-polyacrylamide gels. Proteins were transferred to polyvinylidene difluoride membrane using a Mini 
Trans-Blot system (Bio-Rad) before blocking in $1 \times$ Tris-buffered saline with $0.1 \%$ Tween-20 with $5 \%$ skimmed-milk for $1 \mathrm{~h}$ at 20 $25^{\circ} \mathrm{C}$. Membranes were then incubated with rabbit anti-REST (Millipore, $1: 2000)$, mouse anti-actin $(1: 2000$; Abcam, UK) or rabbit anti-RNA Polymerase II (PolIIS5, $1: 2000$; Abcam) followed by goat anti-rabbit horseradish peroxidase (1:7500; Santa Cruz) or goat anti-mouse horseradish peroxidase (1 : 2000; Dako, Carpinteria, CA, USA) secondary antibodies. Chemiluminescence was performed using Lumi-Light Western Blot Substrate and LumiFilm (Roche) developed using a CompactX4 X-ray developer. Membranes were stripped between detections using a Re-Blot Plus Kit (Chemicon) according to manufacturer's instructions.

\section{Results}

Changes in gene and protein expression following decoy ODN delivery in $\mathrm{Hdh}^{109 / 109}$ cells

$H d h^{109 / 109}$ cells provide an ideal model system in which to test the effect of a decoy ODN on REST-mediated gene dysregulation since these cells have reduced BDNF mRNA and protein levels compared with $H d h^{7 / 7}$ cells and show derepression of Bdnf and other REST target genes following infection with a dominant-negative REST (DN : REST) adenovirus (Zuccato et al. 2007). Compared with wild-type $H d h^{7 / 7}$ cells, mutant $H d h^{109 / 109}$ cells displayed increased nuclear localisation of REST by both immunofluorescence (Fig. 1a) and nuclear and cytoplasmic fraction western blotting (Fig. 1b) as previously reported (Zuccato et al. 2003). To target REST function in this system, we designed a decoy ODN $(++D)$ that contained a canonical RE1 sequence, which can be bound by the REST DNA-binding domain. We additionally designed a negative control decoy ODN (--D) carrying a non-canonical RE1 site that cannot be bound by REST (Bruce et al. 2004). Both sense and antisense oligonucleotides were synthesised with phosphorothiolate bases at their $5^{\prime}$-ends to protect against exonuclease action. To demonstrate the efficacy and specificity of the REST decoy ODN, we investigated the expression of three REST target genes: Bdnf, Chrm4 and Syn1 together with $\beta$-actin. Bdnf, Chrm4 and Synl have all been shown to be aberrantly repressed by REST in $H d h^{109 / 109}$ cells (Zuccato et al. 2007), whereas $\beta$-actin is neither bound nor regulated by REST.

$H d h^{109 / 109}$ cells were transfected with the REST decoy ODN (++D) or non-canonical decoy (--D) and expression of Bdnf, Syn1, Chrm4 and Rest was analysed by qPCR in untransfected $H d h^{7 / 7}$ and $H d h^{109 / 109}$ cells and in $H d h^{109 / 109}$ cells transfected with decoy ODNs $48 \mathrm{~h}$ following transfection (Fig. 1c). Bdnf, Syn1 and Chrm4 all showed significantly reduced expression in $H d h^{109 / 109}$ compared with control $H d h^{7 / 7}$ cells. $H d h^{109 / 109}$ cells transfected with REST decoy ODN showed significant up-regulation of all three REST target genes compared with untransfected cells and compared with cells transfected with --D (Fig. 1c). --D transfected cells showed no significant change in expression of Bdnf, Syn1 or Chrm4 compared with untransfected cells ( $p$-value $=0.11,0.12$ and 0.05 , respectively). REST and actin expression were unaltered in all conditions (Fig 1c). The observed level of decoy-mediated de-repression of target genes was comparable with that obtained using DN : REST and Rest shRNA strategies (Figure S1). In addition, rescue of Bdnf gene expression was accompanied by increased Bdnf protein levels as measured by ELISA assays (Fig. 1d). Untransfected $H d h^{109 / 109}$ cells expressed approximately $26 \%$ of $H d h^{7 / 7}$ Bdnf levels but following delivery of REST decoy ODN, Bdnf protein levels were significantly increased to $65 \%$ of wild-type levels (Fig. 1d, ++D) compared with levels following negative decoy (--D) transfection. Our results, therefore, indicate that by attenuating REST binding with modified decoy ODNs, it is possible to de-repress multiple REST target genes and to partially restore Bdnf protein levels in HD cells.

\section{REST occupancy of RE1s following decoy ODN delivery}

To directly test whether treatment with decoy ODNs decreased occupancy of REST at its RE1 targets in the genome, we carried out ChIP-qPCR analysis using anti-REST IgG and primers that flank RE1 sites of the Bdnf, Synl and Chrm 4 genes (Fig. 2). Chromatin from decoy-transfected cells $(++\mathrm{D}$ or --D) was collected $48 \mathrm{~h}$ post-transfection, similar to gene and protein expression analyses described above earlier, since this time-point showed the highest level of efficacy in rescue of target gene expression (data not shown). REST occupancy was assessed at three REST target gene RE1s: Bdnf, Syn 1 and Chrm4 (Fig. 2). Higher levels of REST occupancy were seen at all three loci in untransfected $H d h^{109 / 109}$ cells compared with $H d h^{7 / 7}$ cells, consistent with the higher levels of REST seen in $H d h^{109 / 109}$ cells. No change in REST occupancy was detected in cells transfected with the negative decoy (Fig. 2, -D). However, following delivery of the Rest RE1 decoy ODN, REST occupancy was significantly decreased at all three loci in $H d h^{109 / 109}$ cells to essentially wild-type $\left(H d h^{7 / 7}\right)$ levels (Fig. 2,++D). We again performed parallel experiments using DN : REST and Rest shRNA for comparison and showed that RE1 decoy ODN was equally as effective at decreasing REST recruitment to these RE1s (Figure S2). Therefore, modified decoy ODNs that contain an RE1 consensus binding site are able to sequester REST protein from target RE1 sites in the genome, resulting in decreased Rest occupancy at several target genes, including $B d n f$.

\section{Changes to histone acetylation of RE1s following decoy delivery}

Transcriptionally active or transcriptionally silent chromatin states are accompanied by specific histone amino-terminal modifications conferring distinct epigenetic signatures (for review, see Jenuwein and Allis 2001). Numerous studies have demonstrated that REST is able to maintain low levels of 
(a)

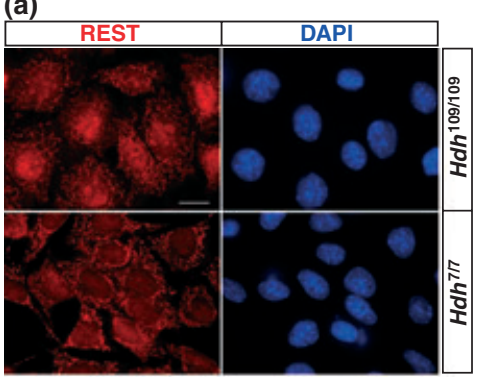

(b)

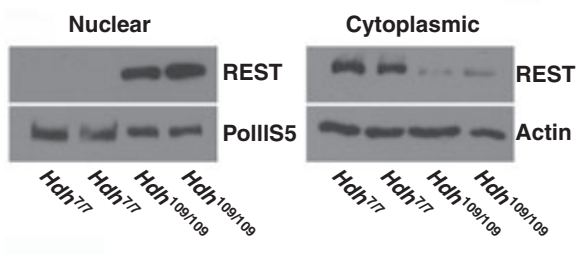

(c)

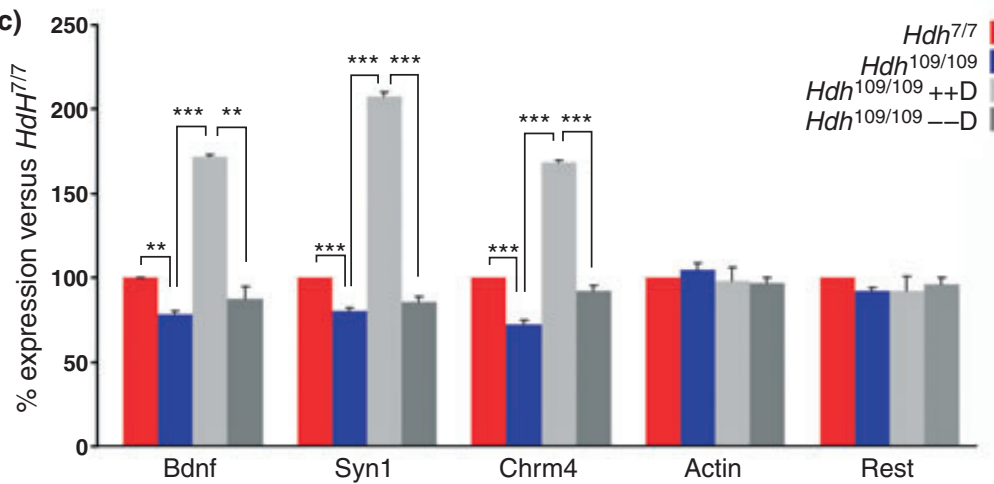

(d)

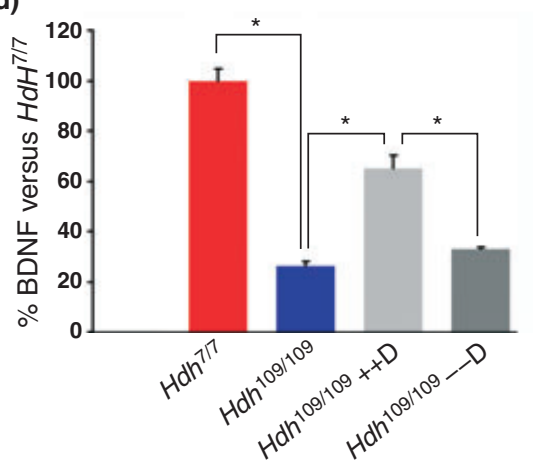

Fig. 1 De-repression of repressor element 1 (RE1) silencing transcription factor (REST) targets and rescue of brain-derived neurotrophic factor (BDNF) protein levels using RE1 decoy oligonucleotides (ODNs). (a) Localisation of REST protein in $\mathrm{Hdh}^{7 / 7}$ and $\mathrm{Hdh}^{109 / 109}$ cells visualised by immunofluorescence (using anti-REST antibody) and (b) western blot of nuclear and cytoplasmic protein fractions (using anti-REST antibody for both and either anti-RNA polymerase II serine 5 or anti-actin for loading controls respectively). REST is much more highly localised to the nucleus in $\mathrm{Hdh}^{109 / 109}$ cells compared with $\mathrm{Hdh}^{7 / 7}$ cells. (c) Bdnf, Syn1 and Chrm4 mRNA levels were measured in $\mathrm{Hdh}^{7 / 7}$ and $\mathrm{Hdh}^{109 / 109}$ cells following delivery of REST decoy ODN $(++D)$ or negative decoy ODN (--D). All three show reduced

histone $\mathrm{H} 3$ lysine 9 acetylation (H3K9ac), an epigenetic marker associated with active chromatin (Dhalluin et al. 1999), at the promoter region of target genes via recruitment of histone deacetylase (HDAC) activity (Grimes et al. 2000; Roopra et al. 2000). To better understand the mechanisms of REST silencing and repression in $H d h^{7 / 7}$ and $H d h^{109 / 109}$ cells, we looked at H3K9ac levels around the RE1s in response to

expression in $\mathrm{Hdh}^{109 / 109}$ compared with $\mathrm{Hdh}^{7 / 7}$ and were increased beyond $\mathrm{Hdh}^{7 / 7}$ levels in $\mathrm{Hdh}^{109 / 109}$ transfected with ++D compared with untransfected cells and cells transfected with --D. Data are expressed as percentage of expression relative to $\mathrm{Hdh}^{7 / 7}$ cell levels and represent the average of three independent experiments. (d) Bdnf protein levels were determined by ELISA on lysates from untransfected $\mathrm{Hdh}^{7 / 7}$ and $\mathrm{Hdh}^{109 / 109}$ cells and $\mathrm{Hdh}^{109 / 109}$ cells transfected with $++\mathrm{D}$ or $--\mathrm{D}$. Data are the average of two independent experiments. In (a) scale bar $=20 \mu \mathrm{m}$. For (b) and (c) ${ }^{*} p \leq 0.05,{ }^{* *} p \leq 0.01$, ${ }^{* * *} p \leq 0.001$ [using Student's $t$-test in (b) and one-way ANOVA in (c)] and error bars show SDs.

changing REST occupancy using our decoy strategy. Figure 3 shows ChIP-qPCR analysis of H3K9ac levels of REST target genes in untransfected $H d h^{7 / 7}$ and $H d h^{109 / 109}$ cells and $H d h^{109 / 109}$ cells $48 \mathrm{~h}$ following delivery of decoy ODNs $(++\mathrm{D}$ or $--\mathrm{D})$. Bdnf and Chrm4 showed more than twofold and Syn 1 almost 1.8-fold lower enrichments of $\mathrm{H} 3 \mathrm{~K} 9 \mathrm{ac}$ in $H d h^{109 / 109}$ cells compared with $H d h^{7 / 7}$ cells (Fig. 3, all 


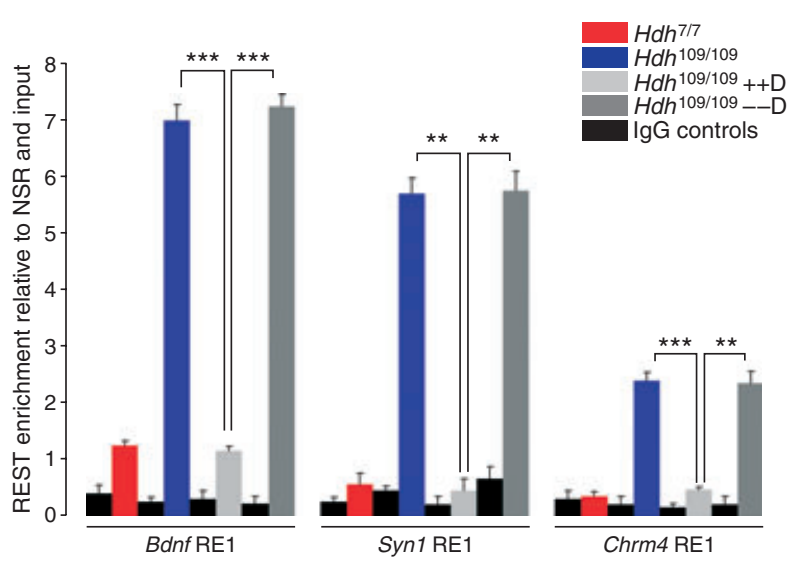

Fig. 2 Repressor element 1 (RE1) silencing transcription factor (REST) occupancy in response to decoy delivery. Chromatin immunoprecipitation (ChIP)-quantitative PCR (qPCR) analysis at three known RE1 sites in untransfected wild-type $\left(\mathrm{Hdh}^{7 / 7}\right)$ and mutant $\left(H d h^{109 / 109}\right)$ cells and in mutant cells following delivery of canonical RE1-containing decoy $(++D)$ or a non-REST binding RE1containing decoy (--D). REST occupancy of the Bdnf, Syn1 and Chrm4 RE1 loci was decreased in $\mathrm{Hdh}^{109 / 109}$ cells transfected with $++D$ compared with untransfected cells and those transfected with --D. Data show REST enrichment relative to both enrichment at a non-specific region that does not contain an RE1 site and to input levels and represent the average of three independent experiments, error bars show SDs, ${ }^{* *} p \leq 0.01,{ }^{* * *} p \leq 0.001$ (using Student's $t$ test).

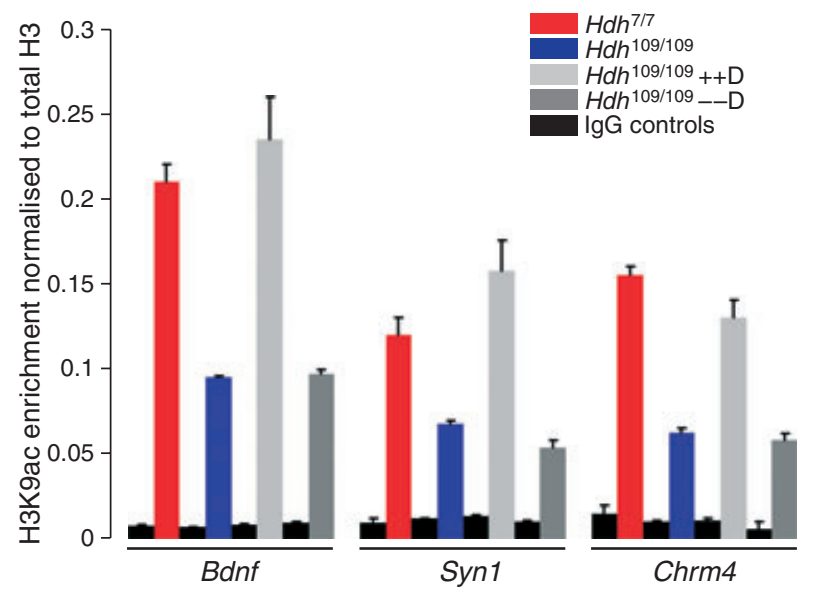

Fig. 3 Effect of decoy delivery on histone $\mathrm{H} 3$ acetyl-lysine 9 (H3K9ac) levels at repressor element 1 (RE1) sites. Chromatin immunoprecipitation (ChIP)-quantitative PCR (qPCR) analysis at three known RE1 loci in untransfected wildtype $\left(\mathrm{Hdh}^{7 / 7}\right)$ and mutant $\left(\mathrm{Hdh}^{109 / 109}\right)$ cells and in mutant cells after delivery of canonical RE1-containing decoy $(++D)$ or a negative decoy $(--D)$. H3K9ac enrichments in $++D$ transfected $\mathrm{Hdh}^{109 / 109}$ cells at Bdnf, Syn1 and Chrm4 RE1s were increased to wild-type levels $\left(\mathrm{Hdh}^{7 / 7}\right)$. H3K9ac enrichment values are shown normalised to total $\mathrm{H} 3$ enrichment to account of any variation in nucleosomal density. $p \leq 0.05$ using Student's $t$-test). We observed a significant increase in $\mathrm{H} 3 \mathrm{~K} 9 \mathrm{ac}$ enrichments at all three loci, to essentially wild-type levels or greater, following REST decoy ODN delivery $(++\mathrm{D})$ compared with untransfected or with negative decoy ODN (--D) transfected cells (Fig. 3, all $p \leq 0.05$ ). Our results indicate that REST is able to maintain low levels of H3K9ac around REST binding sites. We also show that regulation of the local chromatin environment is associated with levels of REST occupancy and reduced H3K9ac observed in $H d h^{109 / 109}$ cells can be restored by the use of modified decoy ODN technology.

RE1-containing decoy ODN interact directly with of REST To demonstrate a direct interaction between REST and the specific REST RE1 decoy ODN we performed an EMSA with a fluorescent (5'-IRD700) labelled decoy ODN (Fig. 4,

$\begin{array}{lllllllll}\text { IR-Decoy (++D) } & + & + & + & + & + & + & + & + \\ \text { Protein } & - & - & + & + & + & + & + & + \\ \text { Antibody } & - & - & - & - & + & + & + & + \\ \text { Decoy (--D) } & - & - & - & - & - & - & + & +\end{array}$

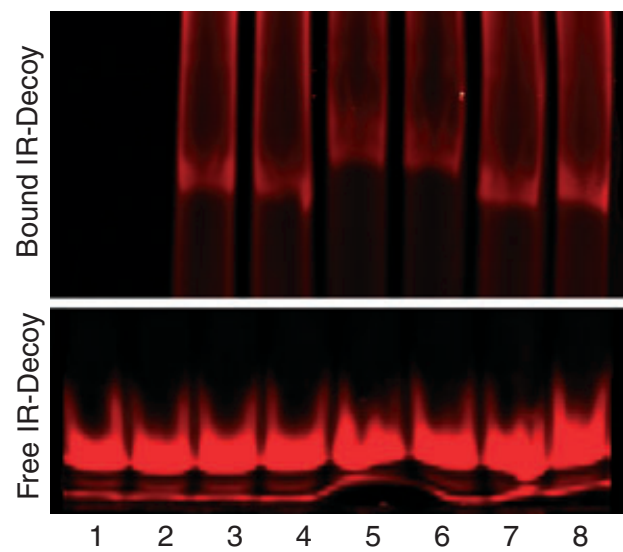

Fig. 4 Decoy oligonucleotides (ODNs) interact directly with repressor element 1 (RE1) silencing transcription factor (REST) protein. The figure shows a fluorescence western blot image with visualisation of an infra-red fluorescence-labelled decoy ODNs containing an RE1-binding site known to bind REST protein (IR-Decoy ++D). The following samples were loaded in duplicate: IR-Decoy ++D alone (lanes 1 and 2); IR-Decoy ++D incubated with HeLa nuclear extract (lanes 3 and 4); IR-Decoy ++D incubated with HeLa nuclear extract and anti-Rest IgG (lanes 5 and 6); HeLa nuclear extract incubated with anti-Rest IgG and excess, non-specific (un-labelled) Decoy ODNs (--D). Free (unbound) IR-Decoy +DD is shown in the bottom panel and alone serves as a negative control (lanes 1 and 2). Incubation of IR-Decoy $++\mathrm{D}$ with HeLa nuclear extract shows an additional band shifted to a higher molecular weight corresponding to a complex of REST protein and IR-Decoy ++D (REST : IR-Decoy). The presence of REST in this complex is confirmed by the super-shifted bands of complexes co-incubated with anti-Rest IgG (lanes 5-6). The REST : IR-Decoy complex is able to form even in the presence of excess quantities of a non-specific, un-labelled decoy containing an RE1 that does not bind to REST (Decoy --D, lanes 7 and 8 ). 
IR-Decoy $++D)$. The IR-Decoy was incubated with or without HeLa cell nuclear extract and the binding reactions were run on a native polyacrylamide gel. Incubation of the decoy with HeLa cell nuclear extract resulted in a specific band shift corresponding to a protein : decoy (REST : IRDecoy) interaction (Fig. 4, lanes 3-4). Inclusion of specific anti-REST IgG resulted in a supershift of the protein : decoy complex (Fig. 4, lanes 5-6) that confirmed that complexes shown by the band shifts in lanes 3 and 4 contained REST protein. Moreover, REST : IR-Decoy complexes were not disrupted when binding reactions were co-incubated with excess negative decoy (Fig. 4, lanes 7-8, --D). The EMSA assays confirm the ability and specificity of the modified REST RE1 decoy ODNs to directly interact with REST protein. Therefore, the observed changes to REST target gene expression, protein expression, REST occupancy and the local chromatin environment in $H d h^{109 / 109}$ cells following introduction of $++\mathrm{D}$ can be attributed to $++\mathrm{D}$ specific sequestration of REST protein.

\section{Discussion}

Use of modified decoy ODNs to abrogate the action of transcription factors is a promising approach to rescuing phenotypic defects because of aberrant transcription factor function. In this work, we have developed a decoy strategy to block the activity of REST based on its binding of the conserved 21bp RE1 (Chong et al. 1995; Schoenherr et al. 1996; Bruce et al. 2004; Johnson et al. 2006). The Bdnf promoter contains an RE1-binding site that has previously been shown to bind to and be regulated by REST both in vitro and in vivo (Zuccato et al. 2001, 2003; Bruce et al. 2004) and recent work has highlighted a critical role for REST in HD through its regulation of BDNF (Zuccato et al. 2007). Here, EMSA we show the ability of a modified double-stranded ODNs that contains a canonical RE1 site to block binding of REST to endogenous binding sites by sequestration of REST protein and to subsequently rescue levels of BDNF mRNA and protein in $H d h^{109 / 109}$ cells. Moreover, using ChIP, we show that there is a concomitant decrease in REST occupancy of the RE1 sites in the $B d n f$ promoter and other target genes. In addition, a concomitant local increase in $\mathrm{H} 3 \mathrm{~K} 9 \mathrm{ac}$ is suggestive of a more open chromatin environment characteristic of activated gene loci. Collectively, these data show that modified decoy ODNs can rescue REST-mediated transcriptional and epigenetic repression in an in vitro model of HD.

\section{Decoy ODN can rescue REST target gene expression including BDNF}

Widespread transcriptional dysregulation is a central pathogenic mechanism in HD and several transcription factors have been implicated in this disease state (Zuccato et al. 2010). Perhaps, the most robust mechanistic link between transcriptional dysfunction and the pathology of HD is provided by REST (Zuccato and Cattaneo 2007). In vitro studies have demonstrated that in normal cells, wild-type Htt sequesters REST in the cytoplasm thereby preventing its binding and repression of target genes (Zuccato et al. 2003). However, in the presence of muHtt, this cytoplasmic complex is disrupted and REST migrates to the nucleus where it can bind to and repress its target genes. Although the initial report focussed on the subsequent repression of the BDNF gene (Zuccato et al. 2003), we have since demonstrated that REST can binds to and repress numerous genes whose expression is altered in the brain and periphery of HD patients, many of which may also be involved in mediating underlying pathological mechanisms (Zuccato et al. 2007; Johnson et al. 2008b; Marullo et al. 2008). Although many studies, including this study have used cell lines to study the transcriptional effects of mutant $\mathrm{Htt}$, it is important to realise that levels of REST tend to be higher in progenitor lines than differentiated neurons. Nevertheless, several studies have indicated the presence of REST protein in the cytoplasm and nucleus of differentiated neurons (Calderone et al. 2003; Kuwabara et al. 2004; Sun et al. 2005; Spencer et al. 2006). Furthermore, one recent study showed that endogenous REST levels were sufficient to repress a RE1-containing BDNF promoter construct in cortical neurons (Hara et al. 2009). Accordingly, we propose that abrogation of REST activity offers a potential strategy to rescue aberrantly repressed REST target genes in differentiated neurons and in HD might ultimately restore globally dysregulated neuronal gene transcription.

Using adenoviral delivery of a dominant-negative form of REST, we have previously shown that in addition to Bdnf, other REST target genes dysregulated in HD, including Syn 1 and Chrm4 (encoding synapsin-1 and the M4 muscarinic receptor, respectively), are also de-repressed in parallel with decreased REST occupancy at their RE1 sites (Greenway et al. 2007; Zuccato et al. 2007; Johnson et al. 2008b). Here, we show that transcription of these genes can also be effectively de-repressed using a decoy ODN strategy, thus offering an exciting alternative to the use of viral or plasmid vectors. Further, the efficacy of decoys relies only on having access to the nuclear transcription factor, whereas dominantnegative constructs based on DNA binding domains need to access the chromatin of target genes.

\section{REST acts as an epigenetic regulator in HD}

One mechanism by which transcription can be impaired is through altered nucleosome dynamics; in this context, histone methylation and acetylation status is closely linked with transcriptional activity, regulating transcription factor access to promoter regions in DNA (Hake et al. 2004; Shogren-Knaak et al. 2006). Recent studies have linked HD transcriptional dysregulation to changes in histone modifications (Sadri-Vakili et al. 2007; Kim et al. 2008), including 
hypo-acetylation of histone $\mathrm{H} 3$ at promoters of downregulated genes in R6/2 mice (Sadri-Vakili et al. 2007). Moreover, treatment with HDAC inhibitors corrects dysregulated gene expression through modification of histone proteins (Ferrante et al. 2003; Sadri-Vakili et al. 2007; Thomas et al. 2008). This may prove to be of therapeutic value in $\mathrm{HD}$, particularly since several HDAC inhibitors are already approved or being validated in clinical trials (for recent review, see Copeland et al. 2010).

REST has been shown to interact with a number of proteins by recruitment of co-repressor platforms via its $\mathrm{N}$ and C-terminal repressor domains (for review, see Ooi and Wood 2007). Many of these proteins are required for REST function via their enzymatic activities that serve to repress or silence gene expression. For example, HDAC1 and HDAC2 are able to repress gene expression by removal of acetyl groups on core histones, including H3K9 (Roopra et al. 2000; Ballas et al. 2001). Similarly REST recruits H3K4 demethylase activity in the form of lysergic acid diethylamide 1, H3K9 methylase activity via G9a and chromatin re-modelling activity, including brahma-related gene 1 and BRAF35 (Battaglioli et al. 2002; Hakimi et al. 2002; Roopra et al. 2004; Lee et al. 2005; Ooi et al. 2006). Therefore, REST is able to both silence and repress neuronal genes by creating a chromatin environment bearing a repressive local epigenetic signature (Greenway et al. 2007).

Since REST repression in other in vitro systems has been shown to be characterised by low levels of H3K9ac (Greenway et al. 2007), we used ChIP to assess changes in H3K9ac following delivery of our decoy ODNs into $H d h^{109 /}$ 109 cells. The low levels of H3K9ac observed around the RE1 sites of REST target genes in $H d h^{109 / 109}$ cells compared with wild-type $H d h^{7 / 7}$ cells is in keeping with the parallel decreases observed in gene expression and the reciprocally increased occupancy of REST at these sites. ++D decoytransfected cells, however, showed significantly increased enrichment of $\mathrm{H} 3 \mathrm{~K} 9 \mathrm{ac}$ at these loci to essentially wild-type levels. These data suggest that REST may have a dual function in target gene repression, including Bdnf, acting both as a transcriptional repressor and epigenetic modulator. This likely occurs through direct binding to its RE1 site and subsequent modification of the surrounding chromatin environment. In this regard, we propose that REST may be considered as much an epigenetic regulator as a transcriptional repressor during the pathological state of HD.

\section{Potential of modified decoy ODN technology in HD}

Over 3000 REST binding sites have been identified in the human and murine genomes, and many target genes encode for proteins that are fundamental for neuronal development and differentiation (Bruce et al. 2004; Johnson et al. 2006). REST is the only known transcription factor able to bind the highly conserved RE1 sequence. Owing to the specificity of this binding and the long $21 \mathrm{bp}$ length of the canonical RE1 sequence, modulation of aberrant REST function associated with HD represents an ideal model for the application of decoy ODN strategies. One of the main limitations of decoy technology has been the rapid degradation of unmodified ODNs by nuclease digestion (Gao et al. 1992; Brown et al. 1994). Different strategies have evolved to render the decoys nuclease-resistant, such as the use of locked nucleic acids (Crinelli et al. 2002) or addition of phosphorothiolated nucleotides (Yang et al. 2002). We chose the latter approach and used a phosphorothiolated decoy ODN based on a canonical RE1 site (REST decoy) as defined by previous bioinformatic and ChIP studies (Bruce et al. 2004; Johnson et al. 2006). Results presented here demonstrate that a phosphorothiolated decoy is sufficiently stable and specific to enable efficient blockade of REST activity. In addition, our results suggest that decoy ODNs are as effective at attenuating REST activity as other previously employed strategies, such as adenoviral delivery of DN : REST or transfection of Rest shRNAs (Greenway et al. 2007; Zuccato et al. 2007 and data not shown). They also avoid the need for large DNA constructs and viral delivery systems and they side-step the issue of potential off-target effects encountered using RNAi technology. Moreover, their ability to directly interfere with transcription factor binding can be directly inferred by using labelled ODNs to serve as probes in EMSAs (Hara et al. 2003; Kubo et al. 2003). With a fluorescence-labelled decoy ODN carrying the canonical RE1 sequence, we were able to directly show the interaction between REST and the decoy and we confirmed the presence of REST in this complex through the use of a specific anti-REST antibody to supershift the protein/DNA complex.

Widespread gene dysregulation is a hallmark of HD and in some cases precedes many of the well-known physical symptoms of the disease (for recent review, see Buckley et al. 2010). The functional products of many of these dysregulated genes have provided new insights into possible mechanisms underlying HD pathology, including the wellknown role of BDNF and concomitant loss of medium spiny neurons (reviewed in Zuccato and Cattaneo 2007). As well as transcriptional dysregulation, it is becoming increasingly clear that epigenetic disruption occurs in HD and, indeed, targeting HDAC activity has become a major platform for HD drug development (Ryu et al. 2006; Kazantsev and Hersch 2007; Kazantsev and Thompson 2008). Many REST functions are attributed to its recruitment of HDAC activity and it is likely that the causative role of REST in HD occurs through an inextricable linkage of transcriptional and epigenetic dysregulation (Buckley et al. 2010). The use of a modified decoy strategy presented here that specifically targets REST function provides a novel and effective means by which it is possible to show effective rescue of both transcriptional and epigenetic effects of REST in an in vitro HD model; this included restoration of Bdnf gene and protein expression and $\mathrm{H} 3 \mathrm{~K} 9 \mathrm{ac}$ levels at its promoter. Modified 
decoys thus offer an attractive alternative to plasmid- or viral-based vectors for interfering with target protein function and provide an ideal strategy to investigate the contribution of REST to HD pathology in well-established mouse models. Perhaps, more intriguingly, since modified ODNs targeting transcription factor function have been shown to be of potential therapeutic value in other disorders (Kubo et al. 2003; De Stefano et al. 2010), they may be useful in future clinical intervention for early-stage HD. The work presented here presents a proof-of-concept that our decoy strategy is capable of rescuing REST-mediated repression of neuronal gene expression a cell line model of HD. Nevertheless, these findings need to be taken forward and the effectiveness of decoys needs to be tested in neuronal cells, where endogenous levels of REST are lower than those found in Hdh cells. Future studies will address this by using in vivo models of HD.

\section{Acknowledgements}

This work is funded by a fellowship from Fondazione Cenci Bolognetti (to CS), the Wellcome Trust (to NJB), the Huntington's Disease Society of America (H.D.S.A., New York) to EC and the Cure HD Initiative (CHDI) Foundation (New York) to EC and NJB. The authors have no competing interests to declare.

\section{Supporting information}

Additional Supporting information may be found in the online version of this article:

Appendix S1. Supplementary Materials and methods.

Figure S1. Reduced REST target gene repression in $H d h^{109 / 109}$ cells using dominant-negative REST or Rest shRNA.

Figure S2. Reduced REST occupancy of RE1 sites in $H d h^{109 / 109}$ cells using dominant-negative REST or Rest shRNA.

As a service to our authors and readers, this journal provides supporting information supplied by the authors. Such materials are peer-reviewed and may be re-organised for online delivery, but are not copy-edited or typeset. Technical support issues arising from supporting information (other than missing files) should be addressed to the authors.

\section{References}

Abrajano J. J., Qureshi I. A., Gokhan S., Zheng D., Bergman A. and Mehler M. F. (2009a) Differential deployment of REST and CoREST promotes glial subtype specification and oligodendrocyte lineage maturation. PLOS ONE 4, e7665.

Abrajano J. J., Qureshi I. A., Gokhan S., Zheng D., Bergman A. and Mehler M. F. (2009b) REST and CoREST modulate neuronal subtype specification, maturation and maintenance. PLOS ONE 4, e7936.

Aharoni-Simon M., Reifen R. and Tirosh O. (2006) ROS-productionmediated activation of AP-1 but not NFkappaB inhibits glutamateinduced HT4 neuronal cell death. Antioxid. Redox Signal. 8, 13391349

Altar C. A., Cai N., Bliven T., Juhasz M., Conner J. M., Acheson A. L., Lindsay R. M. and Wiegand S. J. (1997) Anterograde transport of brain-derived neurotrophic factor and its role in the brain. Nature 389, 856-860.

Bae B. I., Xu H. and Igarashi S. et al. (2005) p53 mediates cellular dysfunction and behavioral abnormalities in Huntington's disease. Neuron 47, 29-41.

Ballas N., Battaglioli E. and Atouf F. et al. (2001) Regulation of neuronal traits by a novel transcriptional complex. Neuron 31, 353365 .

Bates G. P. (2005) History of genetic disease: the molecular genetics of Huntington disease - a history. Nat. Rev. Genet. 6, 766-773.

Battaglioli E., Andres M. E., Rose D. W., Chenoweth J. G., Rosenfeld M. G., Anderson M. E. and Mandel G. (2002) REST repression of neuronal genes requires components of the hSWI.SNF complex. J. Biol. Chem. 277, 41038-41045.

Bithell A., Johnson R. and Buckley N. J. (2009) Transcriptional dysregulation of coding and non-coding genes in cellular models of Huntington's disease. Biochem. Soc. Trans. 37, 1270-1275.

Brown D. A., Kang S. H., Gryaznov S. M., DeDionisio L., Heidenreich O., Sullivan S., Xu X. and Nerenberg M. I. (1994) Effect of phosphorothioate modification of oligodeoxynucleotides on specific protein binding. J. Biol. Chem. 269, 26801-26805.

Bruce A. W., Donaldson I. J., Wood I. C., Yerbury S. A., Sadowski M. I., Chapman M., Gottgens B. and Buckley N. J. (2004) Genome-wide analysis of repressor element 1 silencing transcription factor/ neuron-restrictive silencing factor (REST/NRSF) target genes. Proc. Natl Acad. Sci. USA 101, 10458-10463.

Buckley N. J., Johnson R., Zuccato C., Bithell A. and Cattaneo E. (2010) The role of REST in transcriptional and epigenetic dysregulation in Huntington's disease. Neurobiol. Dis. 39, 28-39.

Calderone A., Jover T. and Noh K. M. et al. (2003) Ischemic insults derepress the gene silencer REST in neurons destined to die. J. Neurosci. 23, 2112-2121.

Canals J. M., Pineda J. R., Torres-Peraza J. F., Bosch M., Martin-Ibanez R., Munoz M. T., Mengod G., Ernfors P. and Alberch J. (2004) Brain-derived neurotrophic factor regulates the onset and severity of motor dysfunction associated with enkephalinergic neuronal degeneration in Huntington's disease. J. Neurosci. 24, 7727-7739.

Cattaneo E., Zuccato C. and Tartari M. (2005) Normal huntingtin function: an alternative approach to Huntington's disease. Nat. Rev. Neurosci. 6, 919-930.

Chae Y. M., Park K. K., Lee I. K., Kim J. K., Kim C. H. and Chang Y. C. (2006) Ring-Sp1 decoy oligonucleotide effectively suppresses extracellular matrix gene expression and fibrosis of rat kidney induced by unilateral ureteral obstruction. Gene Ther. 13, 430439.

Chong J. A., Tapia-Ramirez J. and Kim S. et al. (1995) REST: a mammalian silencer protein that restricts sodium channel gene expression to neurons. Cell 80, 949-957.

Copeland R. A., Olhava E. J. and Scott M. P. (2010) Targeting epigenetic enzymes for drug discovery. Curr. Opin. Chem. Biol. 14, 505-510.

Crinelli R., Bianchi M., Gentilini L. and Magnani M. (2002) Design and characterization of decoy oligonucleotides containing locked nucleic acids. Nucleic Acids Res. 30, 2435-2443.

Dash P. K., Orsi S. A. and Moore A. N. (2005) Sequestration of serum response factor in the hippocampus impairs long-term spatial memory. J. Neurochem. 93, 269-278.

De Stefano D., De Rosa G. and Carnuccio R. (2010) NFkappaB decoy oligonucleotides. Curr. Opin. Mol. Ther. 12, 203-213.

Dhalluin C., Carlson J. E., Zeng L., He C., Aggarwal A. K. and Zhou M. M. (1999) Structure and ligand of a histone acetyltransferase bromodomain. Nature 399, 491-496.

Dunah A. W., Jeong H. and Griffin A. et al. (2002) Sp1 and TAFII130 transcriptional activity disrupted in early Huntington's disease. Science 296, 2238-2243. 
Ferrante R. J., Kubilus J. K. and Lee J. et al. (2003) Histone deacetylase inhibition by sodium butyrate chemotherapy ameliorates the neurodegenerative phenotype in Huntington's disease mice. J. Neurosci. 23, 9418-9427.

Gao W. Y., Han F. S., Storm C., Egan W. and Cheng Y. C. (1992) Phosphorothioate oligonucleotides are inhibitors of human DNA polymerases and RNase H: implications for antisense technology. Mol. Pharmacol. 41, 223-229.

Garriga-Canut M., Schoenike B. and Qazi R. et al. (2006) 2-Deoxy-Dglucose reduces epilepsy progression by NRSF-CtBP-dependent metabolic regulation of chromatin structure. Nat. Neurosci. 9, 1382-1387.

Greenway D. J., Street M., Jeffries A. and Buckley N. J. (2007) RE1 Silencing transcription factor maintains a repressive chromatin environment in embryonic hippocampal neural stem cells. Stem Cells 25, 354-363.

Griesenbach U., Cassady R. L., Cain R. J., duBois R. M., Geddes D. M. and Alton E. W. (2002) Cytoplasmic deposition of NFkappaB decoy oligonucleotides is insufficient to inhibit bleomycin-induced pulmonary inflammation. Gene Ther. 9, 1109-1115.

Grimes J. A., Nielsen S. J. and Battaglioli E. et al. (2000) The corepressor $\mathrm{mSin} 3 \mathrm{~A}$ is a functional component of the REST-CoREST repressor complex. J. Biol. Chem. 275, 9461-9467.

Hake S. B., Xiao A. and Allis C. D. (2004) Linking the epigenetic 'language' of covalent histone modifications to cancer. $\mathrm{Br} . \mathrm{J}$. Cancer 90, 761-769.

Hakimi M. A., Bochar D. A., Chenoweth J., Lane W. S., Mandel G. and Shiekhattar R. (2002) A core-BRAF35 complex containing histone deacetylase mediates repression of neuronal-specific genes. Proc. Natl Acad. Sci. USA 99, 7420-7425.

Hara T., Hamada J., Yano S., Morioka M., Kai Y. and Ushio Y. (2003) CREB is required for acquisition of ischemic tolerance in gerbil hippocampal CA1 region. J. Neurochem. 86, 805-814.

Hara D., Fukuchi M., Miyashita T., Tabuchi A., Takasaki I., Naruse Y., Mori N., Kondo T. and Tsuda M. (2009) Remote control of activity-dependent BDNF gene promoter-I transcription mediated by REST/NRSF. Biochem. Biophys. Res. Commun. 384, 506-511.

Huang C. C., Faber P. W., Persichetti F., Mittal V., Vonsattel J. P., MacDonald M. E. and Gusella J. F. (1998) Amyloid formation by mutant huntingtin: threshold, progressivity and recruitment of normal polyglutamine proteins. Somat. Cell Mol. Genet. 24, 217-233.

Huntington's Disease Collaborative Research Group (1993) A novel gene containing a trinucleotide repeat that is expanded and unstable on Huntington's disease chromosomes. The Huntington's Disease Collaborative Research Group. Cell 72, 971-983.

Jenuwein T. and Allis C. D. (2001) Translating the histone code. Science 293, 1074-1080

Johnson R. and Buckley N. J. (2009) Gene Dysregulation in Huntington's Disease: REST, MicroRNAs and Beyond. Neuromol. Med. 11, 183-199.

Johnson R., Gamblin R. J., Ooi L., Bruce A. W., Donaldson I. J., Westhead D. R., Wood I. C., Jackson R. M. and Buckley N. J. (2006) Identification of the REST regulon reveals extensive transposable element-mediated binding site duplication. Nucleic Acids Res. 34, 3862-3877.

Johnson D. S., Mortazavi A., Myers R. M. and Wold B. (2007) Genomewide mapping of in vivo protein-DNA interactions. Science 316, 1497-1502.

Johnson R., Teh C. H. and Kunarso G. et al. (2008a) REST regulates distinct transcriptional networks in embryonic and neural stem cells. PLoS Biol. 6, e256.

Johnson R., Zuccato C., Belyaev N. D., Guest D. J., Cattaneo E. and Buckley N. J. (2008b) A microRNA-based gene dysregulation pathway in Huntington's disease. Neurobiol. Dis. 29, 438-445.
Kazantsev A. G. and Hersch S. M. (2007) Drug targeting of dysregulated transcription in Huntington's disease. Prog. Neurobiol. 83, 249 259.

Kazantsev A. G. and Thompson L. M. (2008) Therapeutic application of histone deacetylase inhibitors for central nervous system disorders. Nat. Rev. Drug Discov. 7, 854-868.

Kim M. O., Chawla P., Overland R. P., Xia E., Sadri-Vakili G. and Cha J. H. (2008) Altered histone monoubiquitylation mediated by mutant huntingtin induces transcriptional dysregulation. J. Neurosci. 28, 3947-3957.

Kraner S. D., Chong J. A., Tsay H. J. and Mandel G. (1992) Silencing the type II sodium channel gene: a model for neural-specific gene regulation. Neuron 9, 37-44.

Kubo T., Kinjyo N., Ikezawa A., Kambe T. and Fukumori R. (2003) Sp1 decoy oligodeoxynucleotide decreases angiotensin receptor expression and blood pressure in spontaneously hypertensive rats. Brain Res. 992, 1-8.

Kuwabara T., Hsieh J., Nakashima K., Taira K. and Gage F. H. (2004) A small modulatory dsRNA specifies the fate of adult neural stem cells. Cell 116, 779-793.

Lee I. K., Ahn J. D., Kim H. S., Park J. Y. and Lee K. U. (2003) Advantages of the circular dumbbell decoy in gene therapy and studies of gene regulation. Curr. Drug Targets 4, 619-623.

Lee M. G., Wynder C., Cooch N. and Shiekhattar R. (2005) An essential role for CoREST in nucleosomal histone 3 lysine 4 demethylation. Nature 437, 432-435.

Marullo M., Valenza M., Mariotti C., Di Donato S., Cattaneo E. and Zuccato C. (2008) Analysis of the Repressor Element-1 Silencing Transcription Factor/Neuron-Restrictive Silencer Factor Occupancy of Non-Neuronal Genes in Peripheral Lymphocytes from Patients with Huntington's Disease. Brain Pathol. 20, 96-105.

Meller R., Minami M., Cameron J. A., Impey S., Chen D., Lan J. Q., Henshall D. C. and Simon R. P. (2005) CREB-mediated Bcl-2 protein expression after ischemic preconditioning. J. Cereb. Blood Flow Metab. 25, 234-246.

Mori N., Schoenherr C., Vandenbergh D. J. and Anderson D. J. (1992) A common silencer element in the SCG10 and type II Na+ channel genes binds a factor present in nonneuronal cells but not in neuronal cells. Neuron 9, 45-54.

Nucifora Jr. F. C., Sasaki M. and Peters M. F. et al. (2001) Interference by huntingtin and atrophin-1 with cbp-mediated transcription leading to cellular toxicity. Science 291, 2423-2428.

Ooi L. and Wood I. C. (2007) Chromatin crosstalk in development and disease: lessons from REST. Nat. Rev. Genet. 8, 544-554.

Ooi L., Belyaev N. D., Miyake K., Wood I. C. and Buckley N. J. (2006) BRG1 chromatin remodeling activity is required for efficient chromatin binding by repressor element 1-silencing transcription factor (REST) and facilitates REST-mediated repression. J. Biol. Chem. 281, 38974-38980.

Osako M. K., Tomita N. and Nakagami H. et al. (2007) Increase in nuclease resistance and incorporation of NF-kappaB decoy oligodeoxynucleotides by modification of the 3 '-terminus. J. Gene Med. 9, 812-819.

Palm K., Belluardo N., Metsis M. and Timmusk T. (1998) Neuronal expression of zinc finger transcription factor REST/NRSF/XBR gene. J. Neurosci. 18, 1280-1296.

Pfaffl M. W., Georgieva T. M., Georgiev I. P., Ontsouka E., Hageleit M. and Blum J. W. (2002) Real-time RT-PCR quantification of insulinlike growth factor (IGF)-1, IGF-1 receptor, IGF-2, IGF-2 receptor, insulin receptor, growth hormone receptor, IGF-binding proteins 1, 2 and 3 in the bovine species. Domest. Anim. Endocrinol. 22, 91-102.

Roopra A., Sharling L., Wood I. C., Briggs T., Bachfischer U., Paquette A. J. and Buckley N. J. (2000) Transcriptional repression by 
neuron-restrictive silencer factor is mediated via the Sin3-histone deacetylase complex. Mol. Cell. Biol. 20, 2147-2157.

Roopra A., Qazi R., Schoenike B., Daley T. J. and Morrison J. F. (2004) Localized domains of G9a-mediated histone methylation are required for silencing of neuronal genes. Mol. Cell 14, 727-738.

Rubinsztein D. C. and Carmichael J. (2003) Huntington's disease: molecular basis of neurodegeneration. Expert Rev. Mol. Med. 5 , $1-21$.

Ryu H., Lee J., Hagerty S. W., Soh B. Y., McAlpin S. E., Cormier K. A., Smith K. M. and Ferrante R. J. (2006) ESET/SETDB1 gene expression and histone H3 (K9) trimethylation in Huntington's disease. Proc. Natl Acad. Sci. USA 103, 19176-19181.

Sadri-Vakili G., Bouzou B. and Benn C. L. et al. (2007) Histones associated with downregulated genes are hypo-acetylated in Huntington's disease models. Hum. Mol. Genet. 16, 1293-1306.

Schoenherr C. J. and Anderson D. J. (1995) The neuron-restrictive silencer factor (NRSF): a coordinate repressor of multiple neuronspecific genes. Science 267, 1360-1363.

Schoenherr C. J., Paquette A. J. and Anderson D. J. (1996) Identification of potential target genes for the neuron-restrictive silencer factor. Proc. Natl Acad. Sci. USA 93, 9881-9886.

Shogren-Knaak M., Ishii H., Sun J. M., Pazin M. J., Davie J. R. and Peterson C. L. (2006) Histone H4-K16 acetylation controls chromatin structure and protein interactions. Science 311, 844-847.

Spencer E. M., Chandler K. E. and Haddley K. et al. (2006) Regulation and role of REST and REST4 variants in modulation of gene expression in in vivo and in vitro in epilepsy models. Neurobiol. Dis. 24, 41-52.

Stadlbauer T. H., Wagner A. H., Holschermann H., Fiedel S., Fingerhuth H., Tillmanns H., Bohle R. M. and Hecker M. (2008) AP-1 and STAT-1 decoy oligodeoxynucleotides attenuate transplant vasculopathy in rat cardiac allografts. Cardiovasc. Res. 79, 698-705.

Steffan J. S., Kazantsev A. and Spasic-Boskovic O. et al. (2000) The Huntington's disease protein interacts with p53 and CREB-binding protein and represses transcription. Proc. Natl Acad. Sci. USA 97, 6763-6768.

Steiger J. L., Bandyopadhyay S., Farb D. H. and Russek S. J. (2004) cAMP response element-binding protein, activating transcription factor-4, and upstream stimulatory factor differentially control hippocampal GABABR1a and GABABR1b subunit gene expression through alternative promoters. J. Neurosci. 24, 6115-6126.

Sun Y. M., Greenway D. J., Johnson R., Street M., Belyaev N. D., Deuchars J., Bee T., Wilde S. and Buckley N. J. (2005) Distinct Profiles of REST Interactions with Its Target Genes at Different Stages of Neuronal Development. Mol. Biol. Cell 16, 5630-5638.
Thomas E. A., Coppola G. and Desplats P. A. et al. (2008) The HDAC inhibitor $4 \mathrm{~b}$ ameliorates the disease phenotype and transcriptional abnormalities in Huntington's disease transgenic mice. Proc. Natl Acad. Sci. USA 105, 15564-15569.

Trettel F., Rigamonti D., Hilditch-Maguire P., Wheeler V. C., Sharp A. H., Persichetti F., Cattaneo E. and MacDonald M. E. (2000) Dominant phenotypes produced by the HD mutation in STHdh(Q111) striatal cells. Hum. Mol. Genet. 9, 2799-2809.

Yang Y., Tesmer V. M. and Bina M. (2002) Regulation of HIV-1 transcription in activated monocyte macrophages. Virology 299, 256265.

Yao Y., Kubota T., Sato K., Takeuchi H., Kitai R. and Matsukawa S. (2005) Interferons upregulate thymidine phosphorylase expression via JAK-STAT-dependent transcriptional activation and mRNA stabilization in human glioblastoma cells. J. Neurooncol. 72, 217223.

Ye S. M. and Johnson R. W. (2001) Regulation of interleukin-6 gene expression in brain of aged mice by nuclear factor kappaB. J. Neuroimmunol. 117, 87-96.

Zala D., Benchoua A., Brouillet E., Perrin V., Gaillard M. C., Zurn A. D., Aebischer P. and Deglon N. (2005) Progressive and selective striatal degeneration in primary neuronal cultures using lentiviral vector coding for a mutant huntingtin fragment. Neurobiol. Dis. 20, 785-798.

Zuccato C. and Cattaneo E. (2007) Role of brain-derived neurotrophic factor in Huntington's disease. Prog. Neurobiol. 81, 294-330.

Zuccato C. and Cattaneo E. (2009) Brain-derived neurotrophic factor in neurodegenerative diseases. Nat. Rev. Neurol. 5, 311-322.

Zuccato C., Ciammola A. and Rigamonti D. et al. (2001) Loss of huntingtin-mediated BDNF gene transcription in Huntington's disease. Science 293, 493-498.

Zuccato C., Tartari M. and Crotti A. et al. (2003) Huntingtin interacts with REST/NRSF to modulate the transcription of NRSEcontrolled neuronal genes. Nat. Genet. 35, 76-83.

Zuccato C., Belyaev N. and Conforti P. et al. (2007) Widespread disruption of repressor element-1 silencing transcription factor/ neuron-restrictive silencer factor occupancy at its target genes in Huntington's disease. J. Neurosci. 27, 6972-6983.

Zuccato C., Marullo M., Conforti P., MacDonald M. E., Tartari M. and Cattaneo E. (2008) Systematic assessment of BDNF and its receptor levels in human cortices affected by Huntington's disease. Brain Pathol. 18, 225-238.

Zuccato C., Valenza M. and Cattaneo E. (2010) Molecular mechanisms and potential therapeutical targets in Huntington's disease. Physiol. Rev. 90, 905-981. 